\title{
Pharmacological Approaches and Natural Products for Prevention of Chemotherapy-Induced Peripheral Neuropathy - A Review
}

\author{
Marco Cascella ${ }^{1, a^{*}}$, Maria Rosaria Muzio ${ }^{2, b}$ \\ ${ }^{1}$ Division of Anesthesia, Department of Anesthesia and Pain Medicine, Istituto Nazionale Tumori \\ "Fondazione G. Pascale" - IRCCS, Naples, Italy. \\ ${ }^{2}$ Division of Infantile Neuropsychiatry, UOMI-Maternal and Infant Health, AsI NA 3 SUD, \\ Torre del Greco, via Marconi, 66, 80059 Torre del Greco, NA, Italy. \\ a m.cascella@istitutotumori.na.it, ${ }^{b}$ maramuzio@yahoo.it
}

\begin{abstract}
Keywords: Cancer; Chemotherapy; Polyneuropathy; Chemotherapy-induced peripheral neuropathy; Alternative Medicine
\end{abstract}

\begin{abstract}
Chemotherapy-induced peripheral neuropathy (CIPN) is a one of the most common and severe cancer treatment-related adverse effect. It can often cause the stop of the treatment and affects the long-term quality of life of cancer survivors. Unfortunately, there are no effective agent or protocol to prevent with strong evidence of effectiveness this toxicity prevention of CIPN. Thus, CIPN prevention mainly consists of cumulative dose-reduction or lower dose-intensities, especially in higher risk patients. After a brief description of pathophysiology and features of CIPN, the purpose of this study is to analyse the role of standard pharmacological approaches and natural products for prevention of this serious side effect.
\end{abstract}

\section{Introduction}

A wide range of neurologic complications are associated with antineoplastic drug regimens, such as central neurotoxicity conditions, ranging from minor cognitive deficits to encephalopathy with dementia or even coma, and peripheral neurotoxicity. Many chemotherapy drugs including platinum compounds, antitubulins (taxanes, vinca alkaloids, eribulin), proteasome inhibitors (bortezomib), immunomodulatory agents (thalidomide, lenalidomide, pomalidomide), and some of the newer biologics (alemtuzumab, ipilimumab, brentuximab) are known to cause peripheral nerve damage which is also called chemotherapy-induced peripheral neuropathy (CIPN), [1,2]. It is a common treatment-related adverse, affecting up to $48 \%$ of cancer patients who received chemotherapy [3] and worsening the long-term quality of life (QoL) of cancer survivors, too [4]. Indeed, although CIPN is generally a temporary manifestation, in a third of cases it represents a permanent side effect from the chemotherapy treatment employed and paresthesia and/or painful CIPN can also persist from months to years after the end of the anticancer therapy [5]. Unfortunately, there are no effective agents, or protocol, to prevent with strong evidence of effectiveness this serious treatment-related toxicity, and therapeutic strategies are often of limited effectiveness, too. Nevertheless, because prevention seems to be the most effective way of dealing with CIPN, more investigations are directed at identifying any possible pharmacological strategy in both animal models and clinical trials, and this issue also concerns alternative medicines.

After a brief description of pathophysiology and features of CIPN, the purpose of this study is to analyse the role of standard pharmacological approaches and alternative medicines for prevention of this serious side effect.

\section{Pathophysiology and features of chemotherapy-induced peripheral neuropathy}

The precise pathophysiology of CIPN has not yet been defined. Probably, any group of anticancer drugs exhibits a different mechanisms of damage to the peripheral nervous system [6]. For instance, researches have demonstrated a strong affinity of platinum compounds to the deoxyribonucleic acid (DNA) of spinal ganglion cells [7, 8], and the promotion of microtubule polymerization and inhibition of depolymerization leading to inhibition of axonal transport within 
neurons are involved in taxanes neurotoxicity [9]. Furthermore, preclinical studies suggest that peripheral neuropathy due to proteasome inhibitors is probably the result of moderate pathological changes in Schwann cells and myelin, of the axonal degeneration in all three major primary afferent fibers: $\mathrm{A} \beta, \mathrm{A} \delta$, and $\mathrm{C}[10]$, and dorsal root ganglia (DRG) neuron changes, manifested as nuclear and nucleolar atrophy, and/or loss of neurons [11].

Whatever the precise mechanism, neurotoxic chemotherapy agents usually induce a progressive axonal neuropathy, combined or not with DRG neuronal cell body changes, which finally may lead to disabling symptoms and signs. The most common symptoms reported by patients include sensory symptoms of numbness and tingling, followed by burning, shooting, throbbing, and stabbing feelings, felt mainly on hands and feet. Occasionally, CIPN is characterized by severe pain, structuring the painful CIPN, whose management becomes a very hard task. In addition, patients with CIPN may experience motor neuropathy, conditioning rarely the motor nerve function as motor weakness, autonomic dysfunction and even cranial nerve involvement may occur $[12,13]$.

The assessment of CIPN is not the purpose of this work, thus other works on the topic should be consulted [14]. Yet, the effectiveness of nonpharmacologic interventions, such as electrocutaneous nerve stimulation and acupuncture have been evaluated in more specific reviews [15].

\section{Prevention of chemotherapy-induced peripheral neuropathy}

The recently published guidelines from the American Society of Clinical Oncology (ASCO) on the topic, based on a systematic review of 42 randomized controlled trials investigating 18 agents, found that there are no agents that have shown consistent, clinically meaningful benefits for CIPN prevention [16]. Consequently, prevention of CIPN mainly consists of cumulative dosereduction or lower dose-intensities, especially in patients which are at higher risk to develop neurotoxic side effects or in patients which already have neuropathic symptoms due to diabetes mellitus, hereditary neuropathies, or earlier treatment with neurotoxic chemotherapy, as they are more vulnerable for the development of this therapy-related side effect [14]

Data on neuroprotective agents to use for the CIPN prevention are still discussed. Although several agents, such as amifostine, glutathione (GSH) and $\mathrm{N}$-acetylcysteine, as well as the calciumchannel antagonist nimodipine, and calcium and magnesium infusions hold promise as possible neuroprotective factors, clinical data on these drugs have shown a lack of efficacy $[17,18]$. Amifostine has been proposed to prevent taxane-based neuropathy but it has a poor effectiveness and may increase nausea and vomiting [19]. Several randomized clinical trials showed the protective effects and benefits of GSH against platinum-based neurotoxicity and the use of Nacetylcysteine [20], an antioxidant that increases serum GSH concentrations, induces a reduction in incidence and severity of neuropathy and improvements in nerve conduction [21,22]. On the contrary, GSH is not an effective agent in the prevention of taxane-induced CIPN [23]. Moreover, the use of nimodipine in patients with ovarian cancer demonstrated an augmentation of nausea and vomiting and a subsequent poor compliance to the therapy [24]. Calcium and magnesium infusions in patients receiving oxaliplatin combined with 5-fluorouracil and leucovorin for advanced colorectal cancer were initially associated with a marked reduction of neuropathy [25]. However, a more recent large double-blind randomized trial provided strong evidence that this approach was not able to significantly decrease either the acute or persistent neuropathy associated with oxaliplatin [26]. Additionally, not encouraging have been the results obtained by the use of antiseizure drugs, such as carbamazepine and antidepressants, such as venlafaxine. In fact, these drugs seem to have more results in the treatment of CIPN rather than in its prevention [27].

Other nutraceuticals have shown promise for CIPN prevention caused by selective neurotoxic chemotherapeutic agents. For this purpose, Vitamin E was tested together with cisplatin administration [28], Vitamin B6 with hexamethylmelamine [29], and omega-3 fatty acids seem to be efficient neuroprotective agents for the prophylaxis of paclitaxel-induced peripheral neuropathy [30]. Bove et al. [31] demonstrated that after 2 or 4 cycles of cisplatin patients presented 
significantly lower levels of vitamin E than before treatment. However, a phase III study showed that Vitamin E did not appear to reduce the incidence of sensory neuropathy in patients receiving taxanes, platinum compounds, or combination [32]. More encouraging results were obtained with the administration of all-trans retinoic acid in an animal model and patients with lung cancer [33]. Conversely, acetyl-L-carnitine increased CIPN at 24 weeks compared with placebo although no difference was observed at 12 weeks in women undergoing adjuvant breast cancer therapy [34].

New alternative therapies have been considered for prevention of CIPN and there is considerable interest in the prophylactic role of natural products, such as curcumin [35], matricaria chamomilla [36], ginkgo biloba extract [37], and green tea [38]. Among these, curcumin, which is a polyphenol found in the root of Curcuma longa with anti-inflammatory, antioxidant, and antiprotein-aggregate activities, has been shown neuroprotective against several neurological diseases, including Alzheimer's, disease Parkinson's disease, and stroke [39]. Moreover, this natural agent has been proved non-toxic and tolerable even at high doses in humans [40]. In regard to the prophylactic actions on CIPN, in a preclinical study in rats with cisplatin-induced neuropathy, the adinistation of curcumin at the dose of $200 \mathrm{mg} / \mathrm{kg} /$ day given by gavage, ameliorated the sciatic motor nerve conduction velocity - impaired by cisplatin - and prevented the DRG neuronal cell body changes [41]. Although a major problem concerning the use of curcumin is its poor absorption, and thus its bioavailability, many strategies are being developed to go over this limitation [42].

The combination of Asian herbal medicines showed promise as protective agents against CIPN, too. Herbal medicine in Japan is termed as Kampo medicine, which is derived from traditional Chinese medicine. The Kampo medicine goshajinkigan (GJG) is a complex drug containing 10 medicinal herbs with a wide spectrum of pharmacologic actions [43]. The extract product Goshajinkigan is a standardized spray-dried water extract, which comprehends: Rehmanniae Radix, Achyranthis Radix, Corni Fructus, Dioscoreae Rhizoma, Plantaginis Semen, Alismatis Rhizoma, Poria, Moutan Cortex, Cinnamoni Cortex, and Processi Aconiti Radix. Magnesium stearate, lactose, and fructose fatty acid esters are also included as diluents.

GJG is a pharmaceutical drug covered by the Japanese health insurance program and used to treat pain in low extremities, back pain, numbness, blurred vision, dysuria, pollakiuria, and edema. Experimental studies have shown that GJG relieves neurologic symptoms of diabetic peripheral neuropathy such as cold hyperalgesia and mechanical allodynia [44, 45].

About the effects of GJG on prevention of CIPN, several preclinical and clinical investigations have been conducted. Recently, Nishioka et al. [46], in a randomized placebocontrolled multicenter evaluation conducted on patients affected by peripheral neuropathy associated with oxaliplatin therapy, showed that the incidence of grade 3 peripheral neuropathy was significantly lower in patients receiving oral GJG than in those of the control group. This data confirmed previous finding in which GJG showed itself as an effective agent for paclitaxel-induced neuropathy in experimental rodents [47]. Additionally, a phase 2, multicenter, randomized, doubleblind, placebo-controlled trial showed acceptable margins of safety and tolerability and a promising effect in delaying the onset of peripheral neurotoxicity in colorectal cancer patients treated with oxaliplatin and the prophylactic administration of GJG, given orally at the dose of $7.5 \mathrm{~g} /$ day [48]. Another clinical investigation conducted by Bahar and colleagues [49] confirmed that GJG was able to prevent paclitaxel-induced peripheral neuropathy without interfering with the anti-cancer action of paclitaxel. This last finding represents a further proof regarding the safety profile of GJG.

The neuroprotective effects of GJG involve several mechanisms, including the release of dynorphin with the activation of endogenous $\kappa$-opioid receptors to improve numbness or paresthesia, the promotion of nitric oxide production to improve blood supply to the nerves, and the decreased release of transmitter proteins and sensory receptors associated with $\mathrm{C}$-fiber nociceptor [50-53]. Recently, Nakanishi et al. [54] demonstrated that GJG ameliorates allodynia in injury model mice via suppression of tumor necrosis factor-a expression derived from activated microglia. Consequently, GJG is a promising drug also for the treatment of painful neuropathy induced by chemotherapies. Moreover, GJG seems to have other interesting proprieties which could be used in 
oncology. For instance, one of the components of GJG, Poria (Wolfiporia extensa), has shown an antiemetic effect through 5-HT3A receptor inhibition [55]. About the safety of GJG, a recent phase 2, randomized, double-blind, placebo-controlled study concluded that GJG offers an acceptable safety margin and a promising effect in delaying the onset of grade 2 or greater oxaliplatin-induced peripheral neurotoxicity without impairing chemotherapy efficacy [48], confirming previous animal models findings [56].

To date, there are no strong recommendations about the use of specific neuroprotective agents and also the effectiveness of herbal medicines, such as GJG must be better elucidated [57-59]. Nevertheless, standard approaches, including amifostine and antidepressants have had limited efficacy and may themselves induce adverse side-effects, while herbal medicines has shown a better efficacy and safety profile.

\section{Conclusion}

According to the ASCO guidelines, overall no drug can be proposed as a gold standard to prevent CIPN, and this side effect is still difficult to prevent and control without resorting to dose reduction or cessation of chemotherapy treatment. Consequently, new alternative medicines, such as natural products, or the combination of Asian herbal medicines, have been evaluated for their potential therapeutic benefits that can prevent the onset, or limit the progression of CIPN. Despite standard approaches, herbal medicines have shown a better efficacy and safety profile. On these basis, research should explore more about these novel strategies in order to offer more effectiveness drug molecules for the future.

Abbreviations. CIPN: Chemotherapy-induced peripheral neuropathy; DNA: Deoxyribonucleic acid; DRG: Dorsal root ganglia; ASCO: American Society of Clinical Oncology; GJG: Goshajinkigan.

Funding/Financial Support. The authors have no funding to report

\section{References}

[1] C. Parnell, P.J Woll, Principles of cancer treatment by chemotherapy, Surgery (Oxford). 21 (2003) 272-276.

[2] J. Hildebrand, Neurological Adverse Reactions to Anticancer Drugs, Springer, Verlag, 1990.

[3] S. Park et al., Chemotherapy-induced peripheral neurotoxicity: a critical analysis, CA Cancer J. Clin. 63 (2013) 419-437.

[4] E. M. Smith et al., The reliability and validity of a modified total neuropathy score-reduced and neuropathic pain severity items when used to measure chemotherapy-induced peripheral neuropathy in patients receiving taxanes and platinums, Cancer Nurs. 33 (2010) 173-183.

[5] M.A Tanay, J. Armes, E. Ream, The experience of chemotherapy-induced peripheral neuropathy in adult cancer patients: a qualitative thematic synthesis, Eur. J. Cancer Care. (2016).

[6] K. Brzeziński, Chemotherapy-induced polyneuropathy. Part I. Pathophysiology, Contemp. Oncol. 16 (2012) 72-78.

[7] E.S. McDonald et al., Cisplatin preferentially binds to DNA in dorsal root ganglion neurons in vitro and in vivo: a potential mechanism for neurotoxicity, Neurobiol. Dis. 18 (2005) 305313.

[8] L.E Ta et al., Neurotoxicity of oxaliplatin and cisplatin for dorsal root ganglion neurons correlates with platinum-DNA binding, Neurotoxicology. 27 (2006) 992-1002. 
[9] S.M. Swain, J.C. Arezzo, Neuropathy associated with microtubule inhibitors: diagnosis, incidence, and management, Adv. Hematol. Oncol. 6 (2008) 455-467.

[10] G. Cavaletti et al., Bortezomib-induced peripheral neurotoxicity: a neurophysiological and pathological study in the rat, Experimental Neurology. 204 (2007) 317-325.

[11] J.P. Cata et al., Quantitative sensory findings in patients with bortezomib-induced pain, Journal of Pain 8 (2007) 296-306.

[12] A.A. Argyriou, G. Iconomou, H.P. Kalofonos, Bortezomib-induced peripheral neuropathy in multiple myeloma: a comprehensive review of the literature, Blood. 112 (2008) 1593-1599.

[13] A.A Argyriou et al., Chemotherapy-induced peripheral neurotoxicity (CIPN):an update, Crit. Rev. Oncol. Hematol. 82 (2012) 51-77.

[14] M. Cascella, A. Cuomo, D. Viscardi, Pain syndromes associated with cancer therapy, in: M. Cascella, A. Cuomo, D. Viscardi (Eds), Features and Management of the Pelvic Cancer Pain, Springer, Verlag, 2016, pp. 25-62.

[15] G. Franconi et al., A systematic review of experimental and clinical acupuncture in chemotherapy-induced peripheral neuropathy, Evid. Based Complement. Alternat. Med. 2013 (2013).

[16] D.L Hershman et al., Prevention and management of chemotherapy-induced peripheral neuropathy in survivors of adult cancers: American Society of Clinical Oncology clinical practice guideline. J. Clin. Oncol. 32 (2014) 1941-1967.

[17] R. Fernandes et al., Treatment of taxane acute pain syndrome (TAPS) in cancer patients receiving taxane-based chemotherapy-a systematic review, Supportive Care in Cancer. 24(4) (2016) 1583-1594.

[18] F. Hilpert et al., Neuroprotection with amifostine in the first-line treatment of advanced ovarian cancer with carboplatin/paclitaxelbased chemotherapy-a double-blind, placebocontrolled, randomized phase II study from the Arbeitsgemeinschaft Gynakologische Onkologoie (AGO) Ovarian Cancer Study Group, Supportive Care in Cancer. 13(10) (2005) 797-805.

[19] O. Kanat et al., Protective effect of amifostine against toxicity of paclitaxel and carboplatin in non-small cell lung cancer: A single center randomized study, Med. Oncol. 20 (2003) 237245.

[20] P.C. Lin et al., N-acetylcysteine has neuroprotective effects against oxaliplatin-based adjuvant chemotherapy in colon cancer patients: Preliminary data, Supportive Care in Cancer. 14(5) (2006) 484-487.

[21] M. Schmidinger et al., Glutathione in the prevention of cisplatin induced toxicities. A prospectively randomized pilot trial in patients with head and neck cancer and non small cell lung cancer, Wiener Klinische Wochenschrift. 112 (2000) 617-623.

[22] S. Cascinu et al., Neuroprotective effect of reduced glutathione on oxaliplatin-based chemotherapy in advanced colorectal cancer: A randomized, double-blind, placebo controlled trial, J. Clin. Oncol. 20 (2002) 3478-3483.

[23] A. Leal et al., The use of glutathione for prevention of paclitaxel/carboplatin induced peripheral neuropathy: A phase III randomized, double-blind placebo-controlled study, Cancer. 120 (2014) 1890-7.

[24] J. Cassidy et al., Clinical trials of nimodipine as a potential neuroprotector in ovarian cancer patients treated with cisplatin, Cancer Chem. Pharmacol. 41 (1998) 161-166. 
[25] L. Gamelin et al., Prevention of oxaliplatin-related neurotoxicity by calcium and magnesium infusions: A retrospective study of 161 patients receiving oxaliplatin combined with 5fluorouracil and leucovorin for advanced colorectal cancer, Clin. Cancer Res.10 (2004) 40554061.

[26] C.L. Loprinzi et al., Phase III randomized, placebo-controlled, double-blind study of intravenous calcium and magnesium to prevent oxaliplatin-induced sensory neurotoxicity (N08CB/Alliance), J. Clin. Oncol. 32 (2014) 997-1005.

[27] J. P. Durand et al., Efficacy of venlafaxine for the prevention and relief of oxaliplatin-induced acute neurotoxicity: results of EFFOX, a randomized, double-blind, placebo-controlled phase III trial, Ann. Oncol. 23 (2012) 200-205.

[28] A. Pace et al., Vitamin E neuroprotection for cisplatin neuropathy: A randomized, placebocontrolled trial, Neurology. 74(9) (2010) 762-766.

[29] P.H. Wiernik et al., Hexamethylmelamine and low or moderate dose cisplatin with or without pyridoxine for treatment of advanced ovarian carcinoma: A study of the Eastern Cooperative Oncology Group, Cancer Invest. 10 (1992) 1-9.

[30] Z. Ghoreishi et al., Omega-3 fatty acids are protective against paclitaxel-induced peripheral neuropathy: A randomized double-blind placebo controlled trial, BMC Cancer. 12 (2012) 355.

[31] L. Bove et al., A pilot study on the relation between cisplatin neuropathy and vitamin E, J. Exp. Clin. Cancer Res. 20 (2001) 277-280.

[32] L.A. Kottschade et al., The use of vitamin $\mathrm{E}$ for the prevention of chemotherapy-induced peripheral neuropathy: results of a randomized phase III clinical trial, Supportive Care in Cancer. 19(11) (2011) 1769-1777.

[33] Ó. Arrieta et al., Retinoic acid reduces chemotherapy-induced neuropathy in an animal model and patients with lung cancer, Neurology. 77 (2011) 987-995.

[34] D.L. Hershman et al., Randomized double-blind placebo-controlled trial of acetyl-L-carnitine for the prevention of taxane-induced neuropathy in women undergoing adjuvant breast cancer therapy, J. Clin. Oncol. 31 (2013) 2627-2633.

[35] M.S. Al Moundhri et al., The effect of curcumin on oxaliplatin and cisplatin neurotoxicity in rats, J. Med. Toxicol. 9 (2013) 25-33.

[36] A.N.A. Abad et al., Effect of matricaria chamomilla hydroalcoholic extract on cisplatininduced neuropathy in mice, Chin. J. Nat. Med. 9 (2011) 126-131.

[37] H.J. Park et al., Ginkgo biloba extract attenuates hyperalgesia in a rat model of vincristineinduced peripheral neuropathy, Anesth. Analg. 115 (2012) 1228-1233.

[38] J.S. Lee et al., Effect of green tea extracts on oxaliplatin-induced peripheral neuropathy in rats, BMC Complement, Altern. Med. 12(1) (2012) 124.

[39] G.M. Cole, B. Teter, S.A. Frautschy, Neuroprotective effects of curcumin, Adv. Exp. Med. Biol. 595 (2007) 197-212.

[40] J. Epstein, I.R. Sanderson, T.T. Macdonald, Curcumin as a therapeutic agent: the evidence from in vitro, animal and human studies, Br. J. Nutr. 103 (2010) 1545-1557.

[41] S. Agthong, A. Kaewsema, T. Charoensub, Curcumin Ameliorates Functional and Structural Abnormalities in Cisplatin-induced Neuropathy, Exp. Neurobiol. 24 (2015) 139-145.

[42] P. Anand et al., Bioavailability of curcumin: problems and promises, Mol. Pharm. 4 (2007) $807-818$. 
[43] T Kono et al., Goshajinkigan oxaliplatin neurotoxicity evaluation (GONE): a phase 2, multicenter, randomized, double-blind, placebo-controlled trial of goshajinkigan to prevent oxaliplatin-induced neuropathy, Cancer Chemother. Pharmacol. 72 (2013) 1283-1290.

[44] T. Uno et al., Effects of Goshajinkigan on insulin resistance in patients with type 2 diabetes, Diabetes Res. Clin. Pract. 69 (2005) 129-135.

[45] W. Watanabe et al., Long-term effects of goshajinkigan in prevention of diabetic complications: a randomized open-labeled clinical trial, Evidence-based Complementary and Alternative Medicine. 2014 (2014).

[46] M. Nishioka et al., The Kampo medicine, goshajinkigan, prevents neuropathy in patients treated by FOLFOX regimen, Int. J. Clin. Oncol. 16 (2011) 322-327.

[47] Y. Matsumura et al., The prophylactic effects of a traditional Japanese medicine, goshajinkigan, on paclitaxel-induced peripheral neuropathy and its mechanism of action, Molecular Pain. 10 (2014) 61.

[48] T. Kono et al., Goshajinkigan oxaliplatin neurotoxicity evaluation (GONE): a phase 2, multicenter, randomized, double-blind, placebo-controlled trial of goshajinkigan to prevent oxaliplatin-induced neuropathy, Cancer. Chemother. Pharmacol. 72 (2013) 1283-1290.

[49] M.A Bahar et al, Herbal Medicine Goshajinkigan Prevents Paclitaxel-Induced Mechanical Allodynia without Impairing Antitumor Activity of Paclitaxel. Evidence-based Complementary and Alternative Medicine. 2013 (2013).

[50] Y. Omiya et al., Analgesia-producing mechanism of processed Aconiti tuber: role of dynorphin, an endogenous kappa-opioid ligand, in the rodent spinal cord, The Japanese Journal of Pharmacology. 79(3) (1999) 295-301.

[51] A. Gotoh et al., Inhibition mechanism of Gosha-jinki-gan on the micturition reflex in rats, J. Pharm. Sci. 96 (2004) 115-123.

[52] T. Imamura et al., Gosha-jinki-gan reduces transmitter proteins and sensory receptors associated with $\mathrm{C}$ fiber activation induced by acetic acid in rat urinary bladder, Neurourol. Urodyn. 27 (2008) 832-837.

[53] E.K. Joseph et al., Oxaliplatin acts on IB4-positive nociceptors to induce an oxidative stressdependent acute painful peripheral neuropathy, J. Pain. 9 (2008) 463-472.

[54] M. Nakanishi et al., Go-sha-jinki-Gan (GJG) ameliorates allodynia in chronic constriction injury-model mice via suppression of TNF- $\alpha$ expression in the spinal cord, Mol. Pain. 12 (2016).

[55] T. Tai et al., Anti-emetic principles of Poria cocos, Planta Med. 61 (1995) 527-530.

[56] S. Ushio et al., Goshajinkigan reduces oxaliplatin-induced peripheral neuropathy without affecting anti-tumour efficacy in rodents, Eur. J. Cancer. 48 (2012) 1407-1413.

[57] J. Schloss, M. Colosimo, L. Vitetta, Herbal medicines and chemotherapy induced peripheral neuropathy (CIPN): a critical literature review, Crit. Rev. Food. Sci. Nutr. (2015).

[58] E. Oki et al., Preventive effect of Goshajinkigan on peripheral neurotoxicity of FOLFOX therapy (GENIUS trial): a placebo-controlled, double-blind, randomized phase III study, Int. J. Clin. Oncol. 20 (2015) 767-775.

[59] C. Brami, T. Bao, G. Deng, Natural products and complementary therapies for chemotherapyinduced peripheral neuropathy: A systematic review, Crit. Rev. Oncol. Hematol. 98 (2016) 325-334. 